\title{
MINIMAL TOTAL ABSOLUTE CURVATURE FOR ORIENTABLE SURFACES WITH BOUNDARY
}

\author{
BY JAMES H. WHITE
}

Communicated by S. S. Chern, September 22, 1973

Let $M$ be an orientable surface with single smooth boundary curve $C$ which is $C^{2}$ imbedded in Euclidean three-space $E^{3}$. ( $M$ may be thought of as a closed orientable surface with a single disc removed.) Let $M_{\varepsilon}$ be the set of points of $E^{3}$ at a distance $\varepsilon$ from $M . M_{\varepsilon}$ is, of course, for small $\varepsilon$, an imbedded closed surface which is almost everywhere $C^{2}$. Using N. Grossman's [1] adaptation of N. Kuiper's [2] definition, we say that $M$ has minimal total absolute curvature if $M_{\varepsilon}$ is tightly imbedded or has the two piece property, TPP [2].

We announce the following result:

THEOREM. Let $M$ be an orientable surface of genus $g$ with a single smooth boundary curve which is. $C^{2}$ imbedded in $E^{3}$. Then $M$ has minimal total absolute curvature if and only if $M$ has $g=0$ and is a planar disc bounded by a convex curve.

The proof uses a series of integral equations and geometric arguments. The outline is as follows. First, in his paper [1], N. Grossman shows that an orientable surface $M$ of genus $g$ with boundary curve $C$ has minimal total absolute curvature only if the following integral equality holds:

$$
\frac{1}{2 \pi} \int_{M}|K| d A+\frac{1}{2 \pi} \int_{C} \kappa d s=1+2 g,
$$

where $K$ is the Gauss curvature of $M$ and $\kappa$ is the Frenet curvature of the boundary curve $C$ considered as a space curve in $E^{3}$, where $d A$ is the area element of $M$ and $d s$ is the arc element of $C$. Note that the righthand side is the sum of the betti-numbers of $M$ and compare with Kuiper [2] for closed surfaces.

Next, the theorem of Gauss-Bonnet yields

$$
\frac{1}{2 \pi} \int_{M} K d A+\frac{1}{2 \pi} \int_{C} \kappa_{g} d s=1-2 g,
$$

AMS (MOS) subject classifications (1970). Primary 53A05; Secondary 58E99. 
where $\kappa_{g}$ is the geodesic curvature of $C$ considered as a curve on the surface $M$.

Adding (1) and (2), we obtain that if $M$ has minimal total absolute curvature,

$$
\frac{2}{2 \pi} \int_{M:\{K>0\}} K d A+\frac{1}{2 \pi} \int_{C}\left(\kappa+\kappa_{g}\right) d s=2,
$$

where the first integral is taken over the points of $M$ where $K>0$.

LEMMA 1. If $M$ has minimal total absolute curvature, then $M$ has TPP.

In [3], L. Rodriguez shows that, if $M$ has TPP,

$$
\frac{1}{2 \pi} \int_{M:\{K>0\}} K d A+\frac{1}{2 \pi} \int_{C}\left(\kappa+\kappa_{g}\right) d s=2 .
$$

Subtracting (4) from (3), we obtain $(1 / 2 \pi) \int_{M:\{K>0\}} K d A=0$, and hence $K \leqq 0$ in the interior of $M$.

LEMMA 2. $K \leqq 0$ in the interior of $M$.

Lemma 3. C is a plane convex curve.

Lemma 3 is proved by using Morse theory and studying the convex hull of $M_{\varepsilon}$.

Lemma 4. $K \equiv 0$ in the interior of $M$.

This follows immediately from Lemmas 2 and 3.

Now Lemma 4 implies $\int_{M}|K| d A=0$, and Lemma 3 implies $(1 / 2 \pi) \int_{C^{\kappa}} d s=1$. Thus, in order for equation (1) to hold $g$ must be zero and $M$ must be a planar disc bounded by a convex curve.

\section{REFERENCES}

1. N. Grossman, Relative Chern-Lashof theorems, J. Differential Geometry 7 (1972), 611-618.

2. N. H. Kuiper, Minimal total absolute curvature for immersions, Invent. Math. 10 (1970), 209-238. MR 42 \#2499.

3. L. Rodriguez, The two-piece-property and relative tightness for surfaces with boundary (xeroxed thesis), Brown University, Providence, R.I.

Department of Mathematics, University of California, los Angeles, CaliFORNIA 90024 